\title{
Statin therapy attenuates growth and malignant potential of human esophageal adenocarcinoma cells
}

\author{
Miral R. Sadaria, MD, Amy E. Reppert, MD, Jessica A. Yu, MD, Xianzhong Meng, MD, PhD, \\ David A. Fullerton, MD, T. Brett Reece, MD, and Michael J. Weyant, MD
}

\begin{abstract}
Objectives: Esophageal adenocarcinoma is an aggressive malignancy generally diagnosed after metastatic spread and currently lacks effective medical therapy. Expression of intracellular adhesion molecule-1 (ICAM-1) is an adverse prognostic indicator in various human tumor cells and contributes significantly to their metastatic potential. Statin therapy reduces circulating ICAM-1 levels in patients with coronary heart disease and is associated with reduction in progression from Barrett esophagus to esophageal adenocarcinoma. We hypothesize that statin therapy may attenuate growth and malignant potential via ICAM-1 expression and nuclear factor-kappa beta activation in human esophageal adenocarcinoma cells.
\end{abstract}

\begin{abstract}
Methods: Verified human esophageal adenocarcinoma cells (FLO-1) were treated with simvastatin, atorvastatin, or pravastatin (10-, 30-, and 50- $\mu \mathrm{mol} / \mathrm{L}$ concentrations). 3-(4,5-dimethylthiazol-2-yl)-2,5-diphenyl tetrazolium bromide viability, 5-bromo-2'-deoxyuridine proliferation, or annexin $\mathrm{V}$ apoptosis assays were performed, or cells were stimulated with tumor necrosis factor-alpha and collected for immunoblotting and flow cytometry.

Results: Simvastatin decreased cell viability and proliferation while increasing apoptosis in a dose-dependent manner $(P<.05)$. Simvastatin attenuated total cellular and cell-surface ICAM-1 expression as well as nuclear factor-kappa beta activation $(P<.05)$. Atorvastatin had mild effects and pravastatin had essentially no effect on growth and metastatic potential of these cells.

Conclusions: We demonstrate that treatment of human esophageal adenocarcinoma cells with simvastatin attenuates growth, by decreasing cell viability, decreasing cell proliferation, and increasing apoptosis, and attenuates metastatic potential, by decreasing expression of key metastatic markers. These findings identify simvastatin as a potential therapeutic and chemopreventive modality to thwart the progression of esophageal adenocarcinoma. (J Thorac Cardiovasc Surg 2011;142:1152-60)
\end{abstract}

Esophageal adenocarcinoma is an aggressive malignancy whose incidence has drastically risen in the United States over the past 3 decades. Within certain populations, a greater than $800 \%$ increase in incidence has been noted during this time period. ${ }^{1,2}$ Although there have been marked improvements in both the medical and surgical treatments of esophageal adenocarcinoma, most patients continue to die of metastatic disease. ${ }^{3}$ Thus, given the dismal prognosis associated with a diagnosis of esophageal adenocarcinoma, establishing and developing improved therapeutic and chemopreventive treatment modalities is imperative. ${ }^{4}$

From the Division of Cardiothoracic Surgery, Department of Surgery, University of Colorado at Denver and Health Sciences Center, Aurora, Colo.

Funding provided by University of Colorado Academic Enrichment Funds.

Disclosures: Authors have nothing to disclose with regard to commercial support.

Read at the 91st Annual Meeting of The American Association for Thoracic Surgery, Philadelphia, Pennsylvania, May 7-11, 2011.

Received for publication May 6, 2011; revisions received July 26, 2011; accepted for publication Aug 3, 2011.

Address for reprints: Michael J. Weyant, MD, Assistant Professor of Surgery, University of Colorado at Denver and Health Sciences Center, Department of Surgery, Division of Cardiothoracic Surgery, Academic Office One, Room 6602, 12631 East 17th Ave, C310, Aurora, CO 80045 (E-mail: Michael.Weyant@UCDenver.edu). $0022-5223 / \$ 36.00$

Copyright $(2011$ by The American Association for Thoracic Surgery doi:10.1016/j.jtcvs.2011.08.004
Expression of intracellular adhesion molecule-1 (ICAM-1) is an adversely associated prognostic indicator in various human cancers, including human esophageal adenocarcinoma. ${ }^{5,6}$ ICAM-1 is an adhesion molecule believed to play a critical role in transendothelial tumor cell migration, ${ }^{7}$ and the ability of the tumor cells to spread via ICAM-1 expression is believed to significantly contribute to their metastatic potential. ICAM-1 expression is primarily mediated by translocation of nuclear factor kappa beta $(\mathrm{NF}-\kappa \mathrm{B})$ into the nucleus of the cell. ${ }^{5,8,9}$ Furthermore, extracellular factors are associated with poor prognosis and found readily in the tumor microenvironment, such as tumor necrosis factor-alpha $(\mathrm{TNF}-\alpha),{ }^{10}$ which promotes the expression of ICAM- 1 via the NF- $\kappa$ B pathway. This specific pathway has been associated with cancer invasion., Further supporting the importance of ICAM-1 in tumor metastasis, both primary esophageal adenocarcinoma and its metastases have been observed to express ICAM-1, whereas normal esophageal tissue does not. ${ }^{6,11}$ Thus, ICAM-1 expression can potentially serve as a marker of the metastatic potential of esophageal adenocarcinoma cells and an indicator of the patient's prognosis.

Statins are competitive inhibitors of 3-hydroxy-3methylglutaryl coenzyme A, the rate-limiting enzyme in the pathway of cholesterol metabolism, and are classically 


\section{Abbreviations and Acronyms \\ ICAM-1 = intracellular adhesion molecule \\ NF- $\kappa \mathrm{B}=$ nuclear factor kappa beta \\ TNF- $\alpha=$ tumor necrosis factor alpha}

prescribed to patients as lipid-lowering agents. However, a growing body of evidence suggests that statins also affect numerous other intercellular processes, including pathways responsible for producing inflammatory mediators and regulating tumor cell growth. ${ }^{12-16}$ In regard to inflammation, statin therapy initiated for the treatment of coronary artery disease not only decreases the risk of morbidity associated with coronary disease but also significantly reduces the level of circulating ICAM- $1 .{ }^{16}$ In relation to cancer cells, statin therapy has been demonstrated to inhibit both growth and proliferation in many tumor cell types in vitro, including esophageal adenocarcinoma. ${ }^{14,15,17-19}$ In esophageal adenocarcinoma cells, statin therapy has been demonstrated to inhibit proliferation and induce apoptosis via inhibition of Ras farnesylation and the extracellular signal-regulated kinase and Akt signaling pathways. ${ }^{15}$ Others have demonstrated simvastatin therapy to induce apoptosis by inhibiting cyclooxygenase- 2 production in esophageal adenocarcinoma cells. ${ }^{19}$ This suggests that statin therapy may be potentially useful in the prevention or treatment of esophageal adenocarcinoma. Given the aforementioned available data on ICAM- 1 expression and statins, we hypothesize that statin therapy may attenuate the growth of human esophageal adenocarcinoma cells by affecting cell viability, proliferation, and apoptosis and also may attenuate their metastatic potential through down-regulation of ICAM- 1 expression and NF- $\kappa$ B activation, as determined by immunoblotting and flow cytometry, suggesting a common pathway between the two.

\section{MATERIALS AND METHODS}

\section{Cell Line and Drug Preparation}

FLO-1 cells, a verified human esophageal adenocarcinoma cell line, ${ }^{20}$ were cultured in Dulbecco's modified Eagle's medium supplemented with $10 \%$ fetal bovine serum, penicillin (100 units $/ \mathrm{mL})$, streptomycin $(100 \mu \mathrm{g} / \mathrm{mL})$, and fungizone $(1.8 \mu \mathrm{g} / \mathrm{mL})$. Cells were cultured incubated at $37^{\circ} \mathrm{C}$ in a humidified atmosphere of $95 \%$ air and $5 \%$ carbon dioxide.

Simvastatin (Sigma-Aldrich, St Louis, Mo) and atorvastatin (Sigma-Aldrich) were dissolved in dimethyl sulfoxide. Pravastatin (Sigma-Aldrich) was dissolved in distilled water. Before each experiment, stock solution of each drug was diluted in serum-reduced medium $(0.5 \%$ fetal bovine serum), and the concentration of dimethyl sulfoxide never exceeded $0.1 \%$ in simvastatin experiments and $0.33 \%$ in atorvastatin experiments.

Recombinant human TNF- $\alpha$ (Sigma-Aldrich) was reconstituted in sterile-filtered distilled water for a final concentration of $100 \mathrm{ng} / \mu \mathrm{L}$. This was aliquoted appropriately and stored at $-20^{\circ} \mathrm{C}$ until future use. At the time of use, the stock solution was thawed and dissolved in phosphatebuffered saline to a working concentration of $4 \mathrm{ng} / \mu \mathrm{L}$.

\section{Cell Treatment}

FLO-1 cells were cultured in full growth medium (10\% fetal bovine serum) at the following densities: $1 \times 10^{4}$ cells per well (96-well plate) for 48 hours for cytotoxicity, viability, and proliferation assays; $5 \times 10^{5}$ cells per well (6-well plate) for 48 hours for apoptosis assay; $3 \times 10^{5}$ cells per well (6-well plate) for 48 hours for immunoblotting; and $4 \times 10^{5}$ cells per well (6-well plate) for 72 hours for flow cytometry. ${ }^{21,22}$ The cells were serum-reduced for the following 24 hours and then treated with either vehicle control (dimethyl sulfoxide for simvastatin and atorvastatin) or various concentrations $(10,30$, or $50 \mu \mathrm{mol} / \mathrm{L})$ of simvastatin, atorvastatin, or pravastatin. ${ }^{15}$ All wells treated with simvastatin and atorvastatin contained equivalent final dimethyl sulfoxide concentrations as the vehicle control. In experiments evaluating cytotoxicity, cell viability, proliferation, and apoptosis, the assays were started 12 hours after statin treatment. In experiments evaluating ICAM-1 or $\mathrm{NF}-\kappa \mathrm{B}$ expression, cells were stimulated 1 hour after statin treatment with $20 \mathrm{ng} / \mathrm{mL}$ of TNF- $\alpha{ }^{21}$ In experiments evaluating ICAM-1 expression, cells were collected appropriately 12 hours after TNF- $\alpha$ stimulation for either immunoblotting or flow cytometry. In experiments evaluating $\mathrm{NF}-\kappa \mathrm{B}$ expression, cells were collected 10 minutes after TNF- $\alpha$ stimulation for immunoblotting.

\section{Cell Viability, Proliferation, and Apoptosis Quantification After Statin Treatment}

To verify that the statin therapy had no cytotoxic effects on the FLO-1 human esophageal adenocarcinoma cells, a lactate dehydrogenase cytotoxicity assay (Cayman Chemical Company, Ann Arbor, Mich) was performed on the treatment media. Lactate dehydrogenase is contained in the cellular cytoplasm and is released into the treatment media with loss of cell membrane integrity, indicating cytotoxicity resulting from stain therapy. None of the concentrations used in this study $(10,30$, or $50 \mu \mathrm{mol} / \mathrm{L})$ of simvastatin, atorvastatin, or pravastatin had cytotoxic effects on the cells.

After completion of the 12-hour statin treatment in 96-well plates, cellular viability was quantified using a commercially available 3-(4,5dimethylthiazol-2-yl)-2,5-diphenyl tetrazolium bromide assay (Roche Diagnostics Corporation, Indianapolis, Ind) according to the manufacturer's instructions. $^{21,22}$

To further delineate whether the changes in cell viability in the treatment groups were from changes in proliferation versus apoptosis, we performed a 5-bromo-2'-deoxyuridine assay 12 hours after statin treatment according to manufacturer's instructions (Roche Diagnostics Corporation). This assay quantifies de novo DNA synthesis as a measure of proliferation. ${ }^{21,22}$

Apoptosis was quantified using a commercially available fluorescein isothiocyanate-conjugated annexin V apoptosis detection kit (BD Biosciences, San Jose, Calif). Twelve hours after statin treatment, the samples were prepared according to the manufacturer's instructions. Each sample was stained with fluorescein isothiocyanate-conjugated annexin V (apoptotic cells) and propidium iodide (necrotic cells). Flow cytometry (FC500 by Beckman Coulter, Brea, Calif) was used to determine the total percent of cells undergoing early and late apoptosis based on the total percent of cells staining positive for annexin $\mathrm{V}$.

\section{Immunoblotting}

Immunoblotting was used to detect ICAM- 1 and activated NF- $\kappa$ B (phosphorylated and total) expression as previously described. ${ }^{21,23}$

\section{Flow Cytometry}

Flow cytometry was used to detect cell-surface ICAM-1 expression as previously described. ${ }^{21}$

\section{Statistical Analysis}

The lactate dehydrogenase cytotoxicity, cell viability (3-[4,5dimethylthiazol-2-yl]-2,5-diphenyl tetrazolium bromide), and 

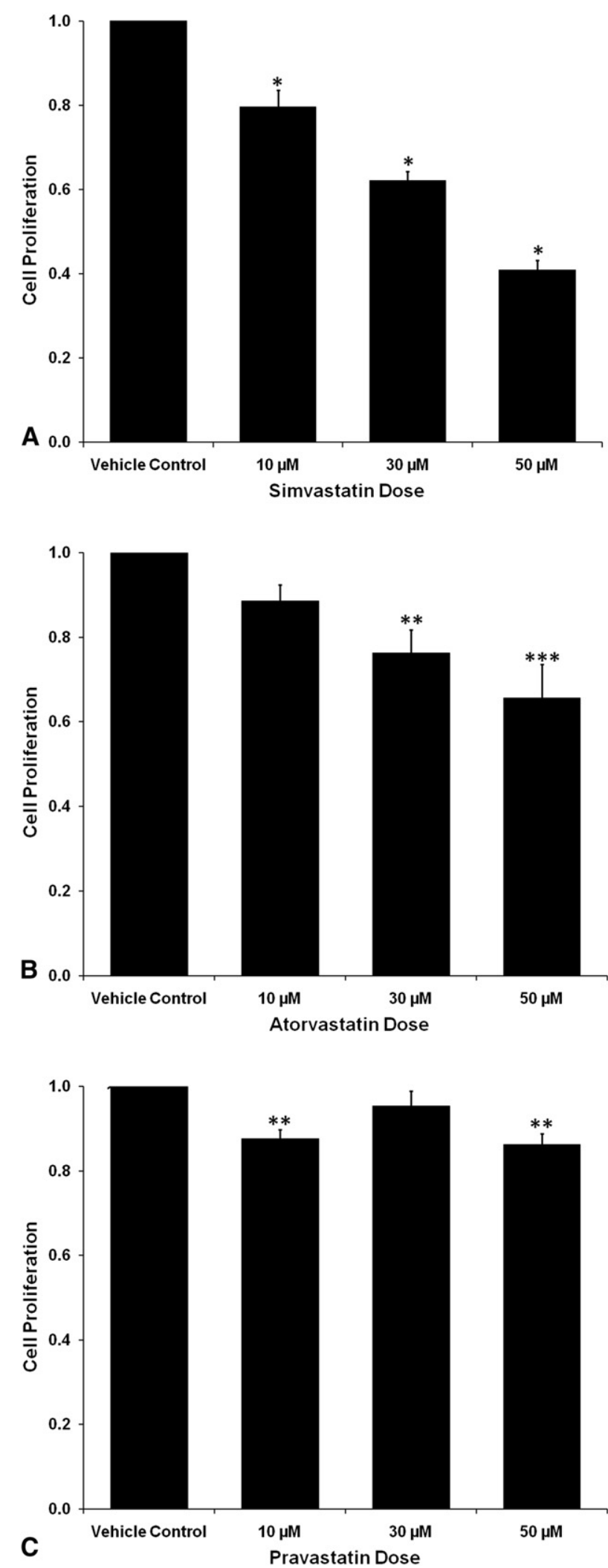

FIGURE 1. Proliferation of human esophageal adenocarcinoma cells (FLO-1) after statin therapy. Simvastatin treatment attenuated FLO-1 cell proliferation at all concentrations (A). Atorvastatin decreased cell prolifer- proliferation (5-bromo- $2^{\prime}$-deoxyuridine) assays were performed in triplicate wells for each experiment. The absorbance value from each well was normalized to vehicle control, giving the vehicle control an adjusted unit value of 1.0. This allowed for comparison of results between experiments and for statistical analysis. The total percentage of cells in each sample undergoing both early and late apoptosis, as indicated by positive annexin $\mathrm{V}$ staining, was used for statistical analysis.

For all immunoblotting results, a density ratio was created by dividing the density of the primary band by the density of the glyceraldehyde-3phosphate dehydrogenase band. The density ratio of each sample was then divided by the density ratio of the vehicle control on the same experimental plate, giving the vehicle control an adjusted unit value of 1.0. By normalizing to the vehicle control, results were able to be compared between experiments, allowing for statistical analysis.

For flow cytometry results, the mean intensity fluorescence of each sample was used to perform statistical analysis.

The phosphorylated and total NF- $\kappa$ B densities were normalized to the glyceraldehyde-3-phosphate dehydrogenase on their respective membranes, accounting for any variation in protein loading. The normalized phosphorylated NF- $\kappa$ B density ratio was then divided by the normalized total NF- $\kappa \mathrm{B}$ density ratio. This adjusted unit was then normalized to the vehicle control to compare results between experimental groups, giving the vehicle control an adjusted unit value of 1.0.

Data are presented as mean \pm standard error. Statistical analysis was performed using analysis of variance with Fisher's least significant difference post hoc test (StatView by SAS Institute, Inc, Cary, NC).

\section{RESULTS}

Cell Viability, Proliferation, and Apoptosis of FLO-1 Human Esophageal Adenocarcinoma Cells After Statin Therapy

Simvastatin, atorvastatin, and pravastatin did not cause any cell necrosis at any of the concentrations used in this study as determined by the lactate dehydrogenase cytotoxicity assay (data not shown).

Treatment with simvastatin attenuated cell viability of FLO- 1 cells by $9.5 \%$ and $16.8 \%$ at the $30-\mu \mathrm{mol} / \mathrm{L}$ and $50-\mu \mathrm{mol} / \mathrm{L}$ concentrations, respectively, compared with the vehicle control $(P<.05, \mathrm{n}=6)$. Treatment with atorvastatin and pravastatin did not affect the cell viability of FLO1 cells $(n=6)$.

FLO-1 cell proliferation was attenuated with simvastatin treatment by $20.4 \%, 37.9 \%$, and $59.0 \%$ at the $10-, 30-$, and $50-\mu \mathrm{mol} / \mathrm{L}$ concentrations, respectively, compared with vehicle control $(P<.0001)$ (Figure $1, A)$. Treatment with atorvastatin attenuated FLO-1 cell proliferation by $23.7 \%$ and $34.4 \%$ at the $30-$ and $50-\mu \mathrm{mol} / \mathrm{L}$ concentrations, respectively $(P<.01)$ (Figure $1, B)$, whereas pravastatin attenuated cell proliferation by $12.3 \%$ and $13.7 \%$ at the 10 - and $50-\mu \mathrm{mol} / \mathrm{L}$ concentrations, respectively, compared with the vehicle control $(P<.005)$ (Figure $1, C)$.

ation at the 30 - and $50-\mu \mathrm{mol} / \mathrm{L}$ concentrations (B), and pravastatin attenuated proliferation of FLO- 1 cells at the 10 - and $50-\mu \mathrm{mol} / \mathrm{L}$ concentrations (C). ${ }^{*} P<.0001$ compared with vehicle control and all concentrations; $* * P<.005$ compared with vehicle control; $* * * P<.01$ compared with vehicle control and $10-\mu \mathrm{mol} / \mathrm{L}$ concentration; $\mathrm{n}=6$. 

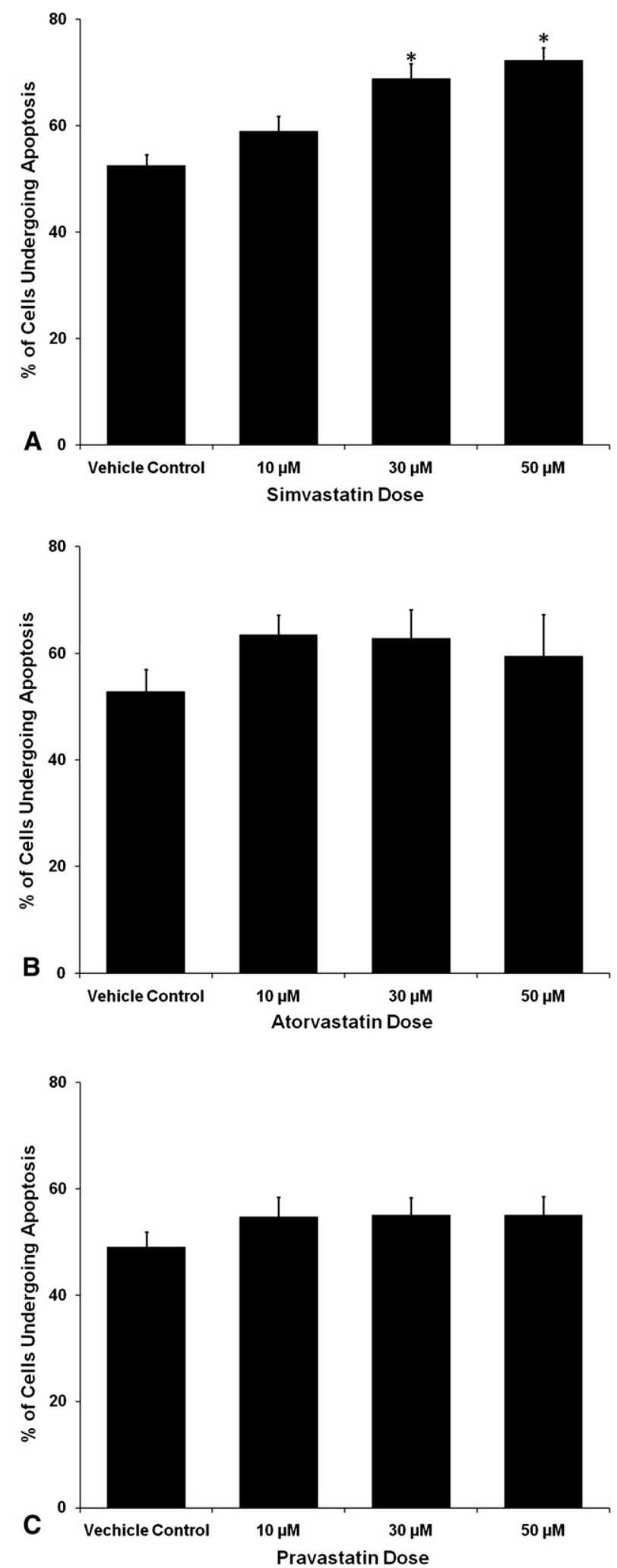

FIGURE 2. Apoptosis of human esophageal adenocarcinoma cells (FLO1) after statin therapy. Simvastatin treatment increased the total percentage of FLO-1 cells undergoing early and late apoptosis at the 30- and 50- $\mu \mathrm{mol} /$ $\mathrm{L}$ concentrations (A), whereas atorvastatin (B) and pravastatin $(\mathrm{C})$ had no
Treatment with simvastatin increased the total percentage of FLO- 1 cells undergoing early and late apoptosis by $16.4 \%$ and $19.8 \%$ at the 30 - and $50-\mu \mathrm{mol} / \mathrm{L}$ concentrations, respectively, compared with the vehicle control $(P<.05)$ (Figure 2, A). Treatment with atorvastatin (Figure 2, B) and pravastatin (Figure 2,C) did not affect the percent of apoptotic FLO-1 cells.

\section{Total Cellular ICAM-1 Expression After Statin Therapy in FLO-1 Human Esophageal \\ Adenocarcinoma Cells}

In FLO-1 human esophageal adenocarcinoma cells, the expression of total cellular and cell-surface ICAM-1 peaks significantly 12 hours after TNF- $\alpha$ administration (data not shown). As previously shown in our laboratory, $20 \mathrm{ng} / \mathrm{mL}$ of TNF- $\alpha$ stimulation significantly increased total cellular and cell-surface ICAM-1 expression in FLO-1 cells compared with vehicle control. ${ }^{21}$

Immunoblotting demonstrated that treatment of FLO-1 human esophageal adenocarcinoma cells with simvastatin and atorvastatin decreased total cellular ICAM-1 expression in a dose-dependent fashion (Figure $3, A$ and $B$ ), whereas pravastatin had no significant effect on total cellular ICAM-1 expression (Figure 3, $C$ and $F$ ). The 30- and 50$\mu \mathrm{mol} / \mathrm{L}$ concentrations of simvastatin produced a $31.3 \%$ and $45.2 \%$ reduction in total cellular ICAM- 1 expression, respectively, compared with the vehicle control $(P<.01)$ (Figure $3, D$ ). The $10-, 30-$, and $50-\mu \mathrm{mol} / \mathrm{L}$ concentrations of atorvastatin produced a $18.5 \%, 23.2 \%$, and $37.7 \%$ reduction in total cellular ICAM-1 expression, respectively, compared with the vehicle control $(P<.05)$ (Figure 3,E).

\section{Cell-Surface ICAM-1 Expression After Statin Therapy in FLO-1 Human Esophageal Adenocarcinoma Cells}

Flow cytometry demonstrated treatment of FLO-1 human esophageal adenocarcinoma cells with simvastatin at $10-, 30-$, and $50-\mu \mathrm{mol} / \mathrm{L}$ concentrations decreased cellsurface ICAM- 1 expression $17.8 \%, 27.5 \%$, and $30.6 \%$, respectively, compared with vehicle control $(P<.05)$ (Figure 4, $A$ and $D$ ). Cells treated with atorvastatin had an $18.2 \%$ and $21.7 \%$ decrease in cell-surface ICAM- 1 expression at the 30 - and $50-\mu \mathrm{mol} / \mathrm{L}$ concentrations, respectively, compared with vehicle control $(P<.05)$ (Figure 4, $B$ and $E$ ). Pravastatin attenuated cell-surface ICAM-1 expression $16.2 \%$ and $13.3 \%$ at the $10-$ and $30-\mu \mathrm{mol} / \mathrm{L}$ concentrations, respectively, compared with vehicle control $(P<.05)$ (Figure 4, $C$ and $F$ ).

effect on the total percentage of cells undergoing apoptosis. $* P<.05$ compared with vehicle control and $10-\mu \mathrm{mol} / \mathrm{L}$ concentration; $\mathrm{n}=6$. 


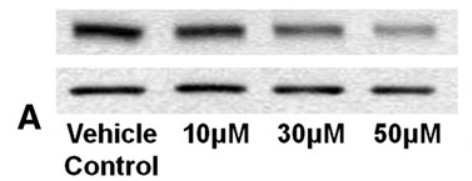

ICAM-1

\section{GAPDH}

Simvastatin Dose Control

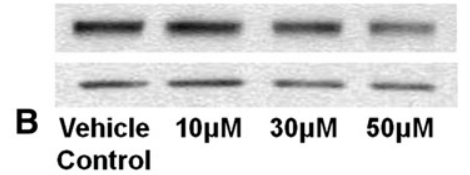

ICAM-1

GAPDH

Atorvastatin Dose Control

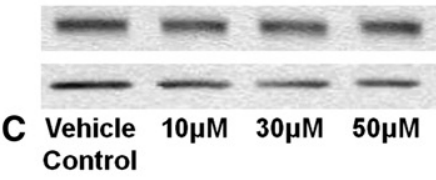

ICAM-1

GAPDH

Pravastatin Dose

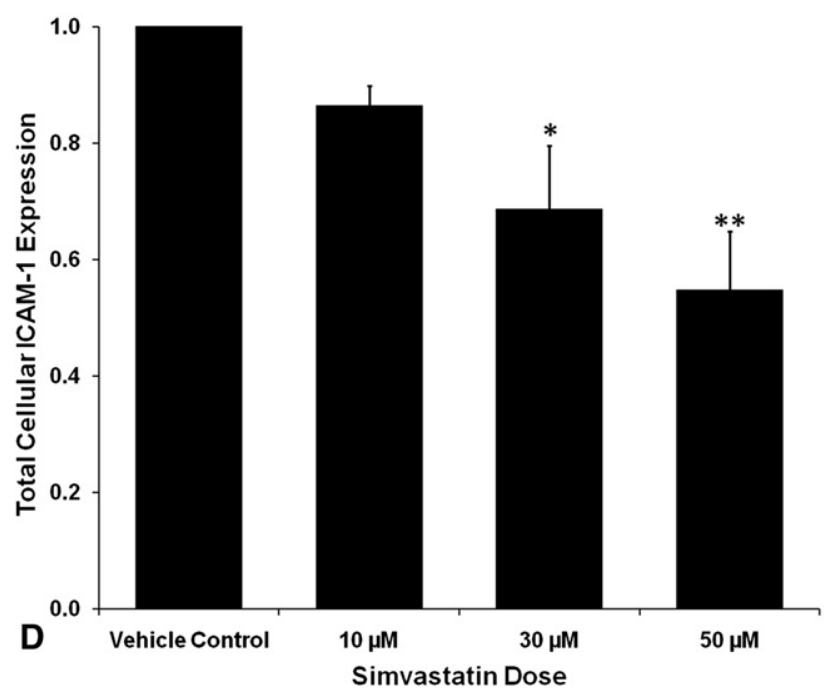

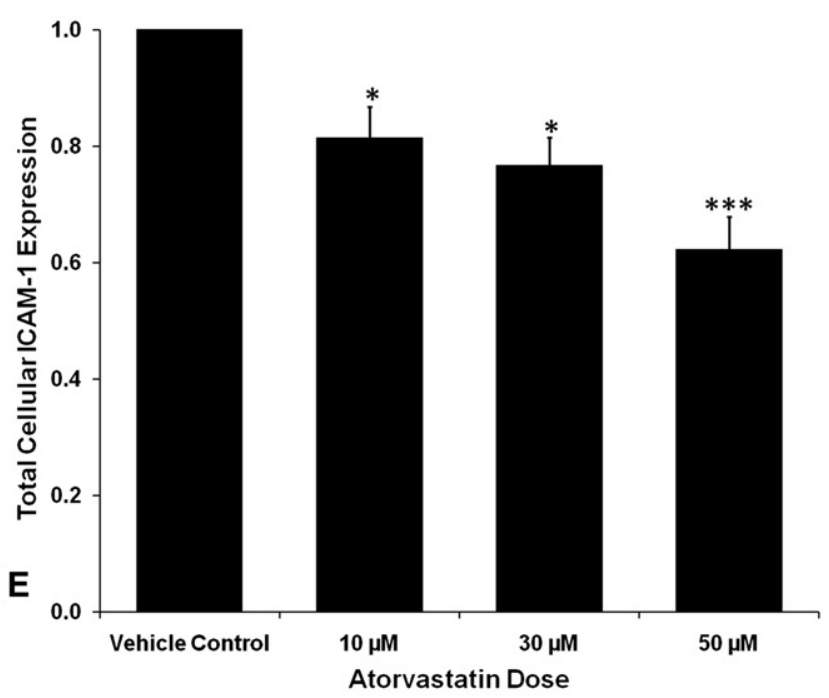

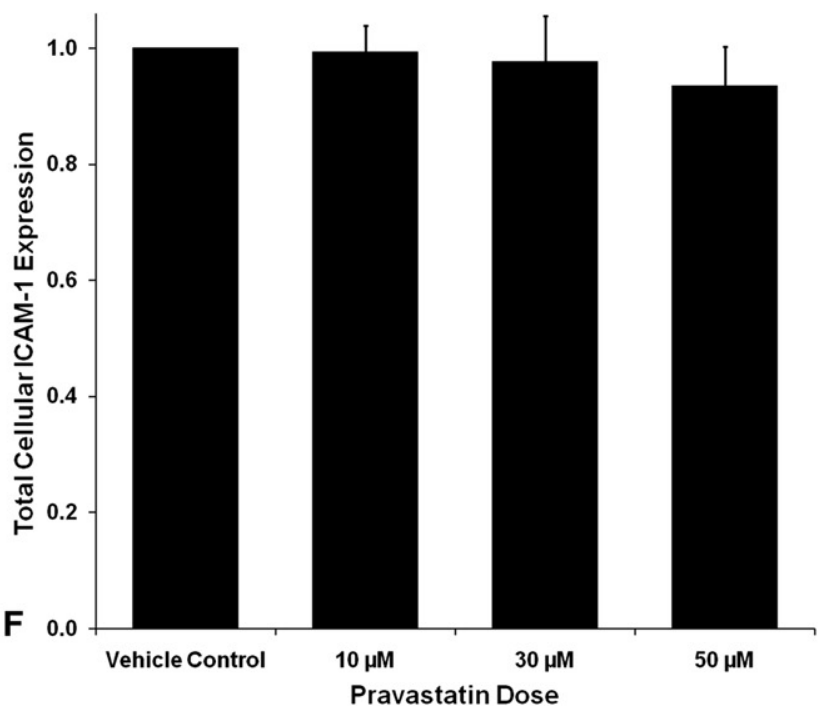

FIGURE 3. Total cellular ICAM-1 expression after statin therapy in human esophageal adenocarcinoma cells ( FLO-1). Representative Western blots demonstrate a dose-dependent attenuation of total cellular ICAM-1 expression in FLO-1 cells when treated with simvastatin (A) and atorvastatin (B), whereas treatment with pravastatin demonstrates no significant attenuation ( $\mathrm{C}$ and $\mathrm{F}$ ). The 30 - and $50-\mu \mathrm{mol} / \mathrm{L}$ concentrations of simvastatin reduced total cellular ICAM-1 expression compared with vehicle control (D), whereas all concentrations of atorvastatin reduced total cellular ICAM-1 expression compared with vehicle control (E). $* P<.01$ compared with vehicle control; $* * P<.01$ compared with vehicle control and $10-\mu$ mol/L concentration $* * * P<.05$ compared with vehicle control and all concentrations; $\mathrm{n}=6 . I C A M-1$, Intracellular adhesion molecule-1; GAPDH, glyceraldehyde-3-phosphate dehydrogenase.

\section{NF- $\kappa$ B Activation After Statin Therapy in FLO-1 Human Esophageal Adenocarcinoma Cells}

In FLO-1 human esophageal adenocarcinoma cells, NF$\kappa \mathrm{B}$ phosphorylation peaked 10 minutes after TNF- $\alpha$ administration (data not shown). When compared with the vehicle control, immunoblotting demonstrated an attenuation of $\mathrm{NF}-\kappa \mathrm{B}$ activation by $17.2 \%, 17.8 \%$, and $30.5 \%$ after treatment of FLO-1 cells with simvastatin at the 10-, 30-, and 50$\mu \mathrm{mol} / \mathrm{L}$ concentrations, respectively $(P<.05)$ (Figure $5, A$ and $D$ ). Cells treated with atorvastatin and pravastatin had no reduction in NF- $\kappa \mathrm{B}$ activation compared with vehicle control (Figure 5, $B, C, E$, and $F$ ).

\section{DISCUSSION}

The aim of this study was to determine the effect of statin therapy on growth and metastatic potential, as determined by the metastatic marker ICAM-1 and its mediator NF$\kappa \mathrm{B}$, of human esophageal adenocarcinoma cells. The results of this study demonstrate that simvastatin treatment of FLO-1 human esophageal adenocarcinoma cells significantly decreased cell viability and proliferation while increasing apoptosis. Additionally, simvastatin treatment attenuated total cellular and cell-surface ICAM-1 expression and levels of activated NF- $\kappa \mathrm{B}$, suggesting a common pathway between simvastatin's effect on ICAM-1 

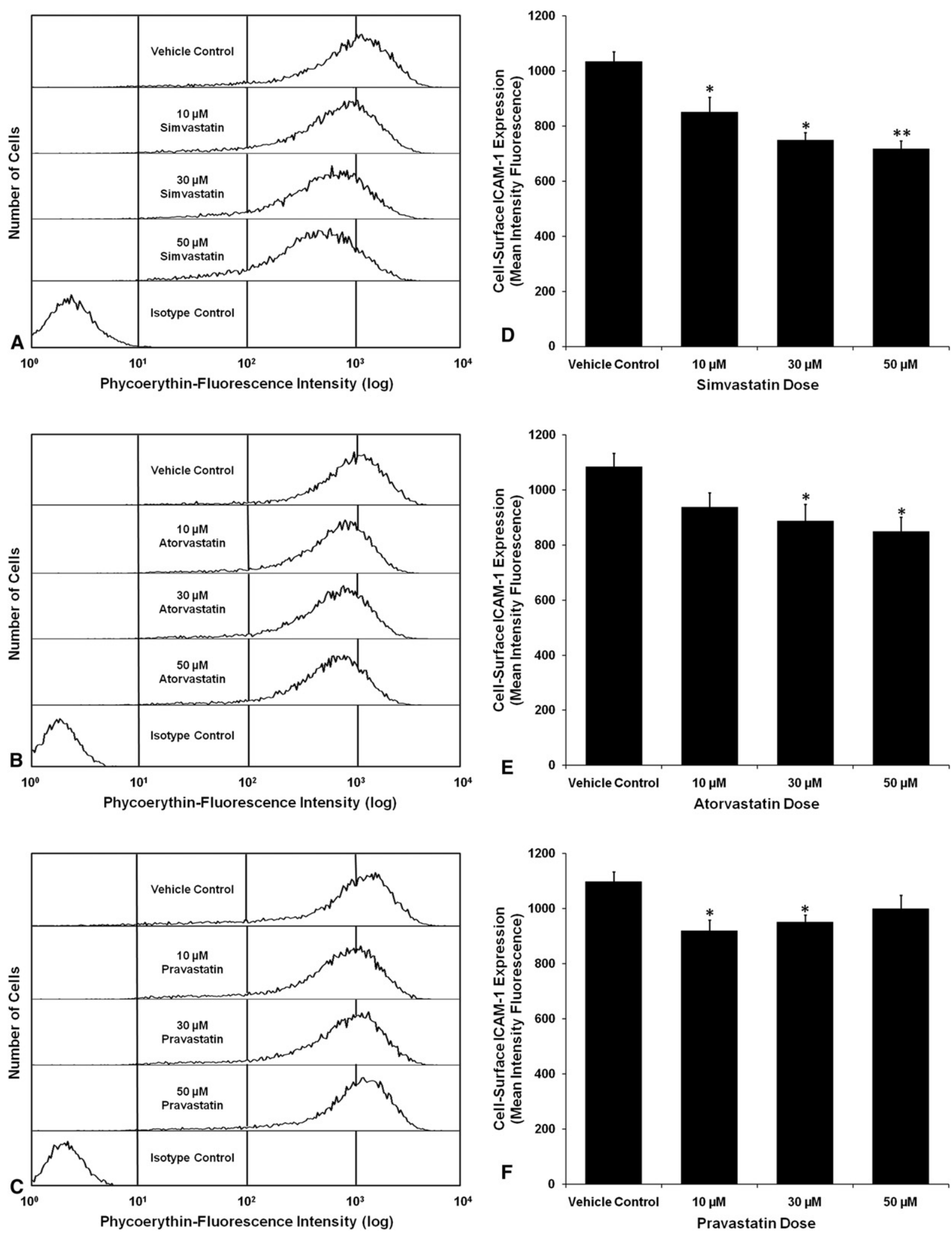

FIGURE 4. Cell-surface ICAM-1 expression after statin therapy in human esophageal adenocarcinoma cells (FLO-1). Representative flow cytometry histograms demonstrate a reduction in mean intensity fluorescence and, thus, cell-surface ICAM-1 expression after treatment of FLO-1 cells with simvastatin (A), atorvastatin (B), and pravastatin (C). Cell-surface ICAM-1 expression decreased with all concentrations of simvastatin (D), 30 and $50 \mu \mathrm{mol} / \mathrm{L}$ of atorvastatin (E), and 10 and $30 \mu \mathrm{mol} / \mathrm{L}$ of pravastatin $(\mathrm{F}) . * P<.05$ compared with vehicle control; $* * P<.05$ compared with vehicle control and $10-\mu$ mol/L concentration; $\mathrm{n}=6$. ICAM-1, Intracellular adhesion molecule-1. 


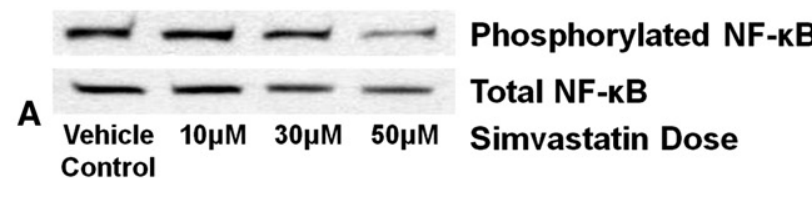

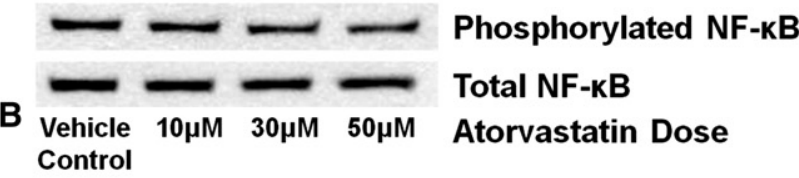
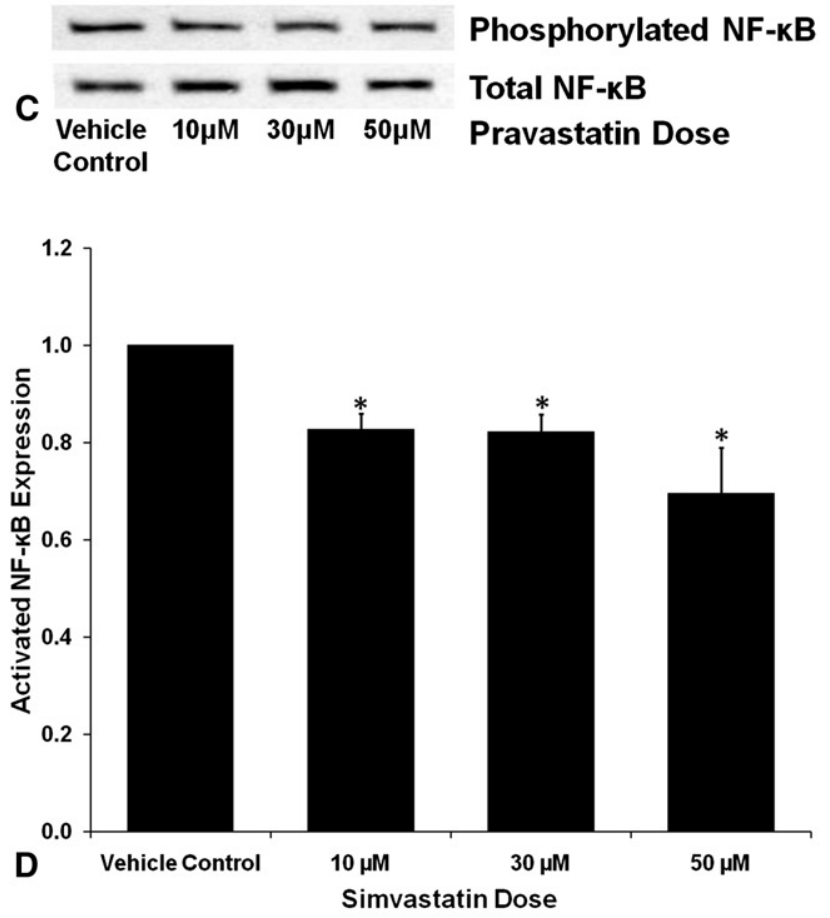
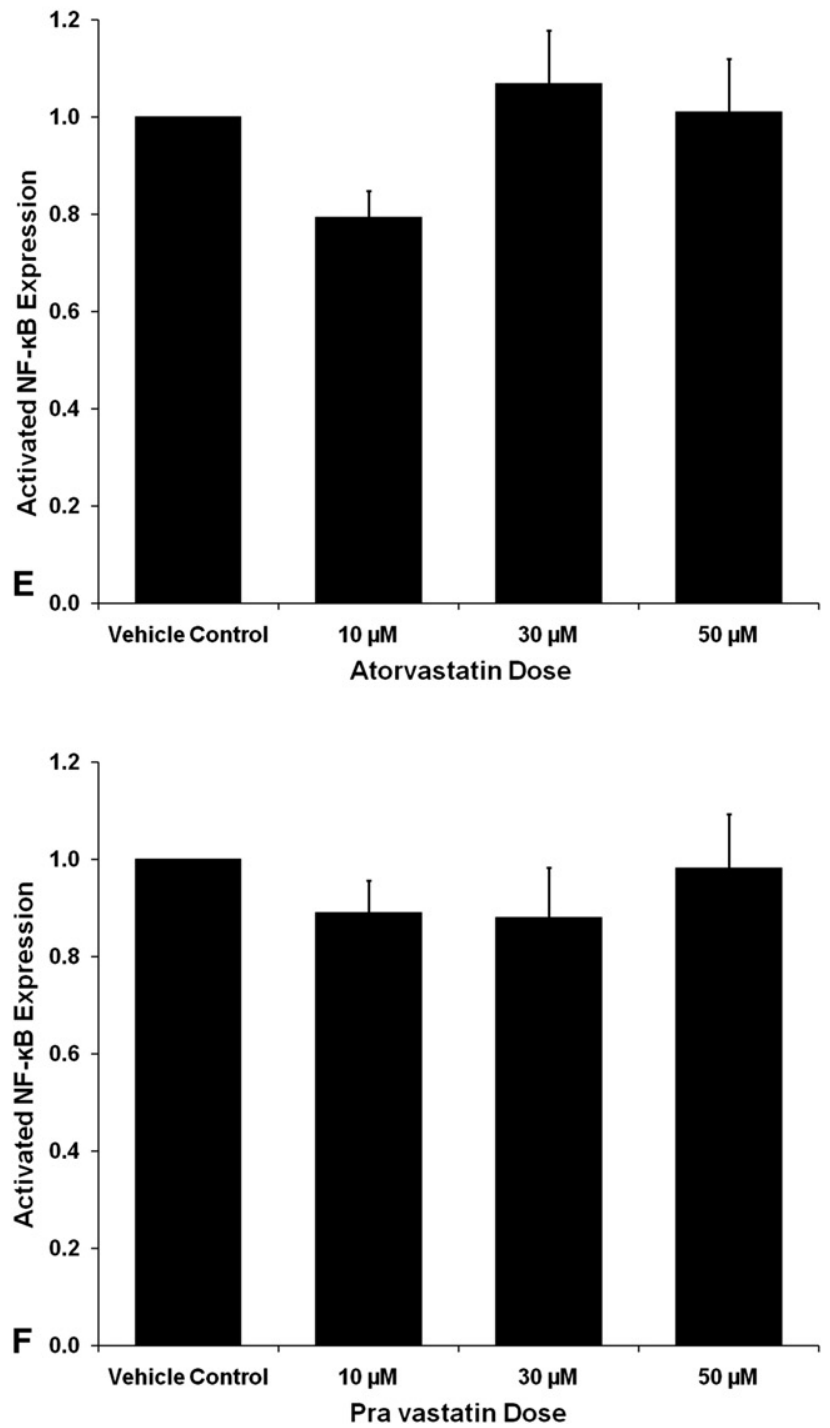

FIGURE 5. NF- $\kappa \mathrm{B}$ activation after statin therapy in human esophageal adenocarcinoma cells (FLO-1). Representative Western blots demonstrate a dosedependent attenuation in NF- $\kappa$ B activation in FLO-1 cells when treated with simvastatin (A), whereas treatment with atorvastatin (B and E) and pravastatin $(\mathrm{C}$ and $\mathrm{F}$ ) demonstrate no significant attenuation when compared with vehicle control. A decrease in NF- $\kappa \mathrm{B}$ activation was demonstrated at all concentrations of simvastatin treatment $(\mathrm{D}) .{ }^{*} P<.05$ compared with vehicle control; $\mathrm{n}=6 . N F-\kappa B$, Nuclear factor kappa beta.

expression and NF- $\kappa \mathrm{B}$ activation. Atorvastatin therapy had milder effects than simvastatin, whereas pravastatin had essentially no effect on the growth and metastatic potential of these cells.

Statin therapy has been studied regarding its relationship to tumorigenesis and its possible role in the treatment of various malignancies. ${ }^{17}$ In vitro and in vivo studies alike have demonstrated the ability of statin therapy to inhibit tumor growth and induce apoptosis in a variety of malignant cells, such as melanoma ${ }^{18}$ and colon cancer. ${ }^{24}$ Additionally, human esophageal adenocarcinoma cells have been demonstrated to be susceptible to treatment with statins. ${ }^{15,19}$ However, only very few reports regarding this phenomenon exist. Ogunwobi and Beales ${ }^{15}$ demonstrated statin therapy to inhibit proliferation and induce apoptosis in OE33-verified esophageal adenocarcinoma cells via inhibition of Ras farnesylation and the extracellular signalregulated kinase and Akt signaling pathways. Furthermore, Konturek, Burnat, and Hahn ${ }^{19}$ demonstrated simvastatin therapy to induce apoptosis and inhibit cyclooxygenase-2 production in OE19-verified esophageal adenocarcinoma cells. In accordance with previously published data, our study demonstrates the ability of statin therapy to attenuate esophageal adenocarcinoma cell viability and proliferation while inducing apoptosis. Interestingly, this effect varies significantly depending on the particular statin used. 
In addition to attenuating their growth, simvastatin inhibits ICAM-1 expression, a marker of the tumor's metastatic potential, as well as reducing levels of activated $\mathrm{NF}-\kappa \mathrm{B}$ in esophageal adenocarcinoma cells, suggesting a common pathway between the two. ${ }^{5,8,9}$ ICAM-1 is a member of a family of adhesion molecules primarily responsible for the migration of leukocytes to the vascular endothelium during inflammation. These leukocytes are then released into the circulation for transport to the injured tissues. Analogous to mechanisms of inflammation, cancer cells travel to sites beyond the primary tumor via ICAM-1. This similarity between cellular movement in response to inflammation and in cancer cell metastasis may provide a link between inflammation and tumor development, further defining a role for anti-inflammatory therapies in the treatment of cancers such as esophageal adenocarcinoma. ${ }^{25}$ Simvastatin's wellestablished anti-inflammatory effects coupled with its ability to attenuate ICAM-1 expression and NF- $\kappa$ B activation identify simvastatin as a potential therapeutic agent to be used synergistically with current chemotherapy in the treatment of esophageal adenocarcinoma.

Although simvastatin has a significant effect on the growth and malignant potential of esophageal adenocarcinoma cells, atorvastatin had a minimal and variable effect and pravastatin had virtually no effect. This inconsistency between the effects of various statin therapies on the human esophageal adenocarcinoma cells may be linked to the differences in their molecular structures, directly affecting their solubility, metabolism, and cellular localization. Specifically, the presence or absence of polar moieties on the largely hydrophobic backbone of satins and variations in the number of these moieties ultimately affect their lipophilicity. The cellular uptake of the lipophilic statins, such as simvastatin and atorvastatin, occurs via passive diffusion, allowing for them to be widely distributed in different tissues and localize within the hydrophobic regions of the cell. In contrast, the cellular uptake of hydrophilic statins, such as pravastatin, occurs via carrier-mediated mechanisms and localizes within polar regions of the cell. ${ }^{26}$ The variations in cellular uptake and areas of localization between statins may directly alter their effects on the cell, thus possibly accounting for the variation in results seen between the lipophilic simvastatin and atorvastatin as compared with the hydrophilic pravastatin. Furthermore, simvastatin is derived from a natural product whereas atorvastatin is a synthetic compound, ${ }^{27}$ possibly accounting for the variability in results obtained between the 2 lipophilic statins.

Beyond merely a mechanistic effect in vitro, a clinical effect has also been noted between statin therapy and progression of the premalignant lesion, Barrett esophagus, to the malignant lesion, esophageal adenocarcinoma. In a nested case-control study conducted in a cohort study of patients diagnosed with Barrett esophagus in the Department of
Veterans Affairs health care system, patients on statin therapy after diagnosis had a significant risk reduction for the development of esophageal adenocarcinoma. Furthermore, a significant trend toward a greater risk reduction with longer duration of statin therapy was noted. ${ }^{28}$ Reaching beyond statin therapy's ability to potentially treat the malignant lesion, a possible role for statin treatment in preventing the conversion of the premalignant lesion to the malignant lesion exists.

Currently, patients with esophageal adenocarcinoma have poor outcomes as a result of metastatic disease, which lacks effective therapeutic or chemopreventive modalities. The results of our present study demonstrate that simvastatin attenuates growth of esophageal adenocarcinoma cells as well as exhibiting the novel effect of reducing their metastatic potential through reduction of total cellular and secreted ICAM-1 expression and NF- $\kappa \mathrm{B}$ activation. These effects do not appear to be globally attributed to all statins, which as stated previously may be due to the physical properties of the drugs themselves. Although this study demonstrates a promising role for statin therapy in the treatment of esophageal adenocarcinoma, examining its effects in only one esophageal adenocarcinoma cell line limits the conclusions of the study. Furthermore, the statin concentrations used in this study are similar to various in vitro anticancer studies, but they are difficult to accurately convert to oral doses owing to factors such as bioavailability, bioactivity, and patient factors. Although these concentrations show no cytotoxicity in cells, clinical trials would have to be conducted to determine patient tolerance in terms of the development of myopathy. Despite these limitations, the findings of this study further our interest in determining the potential role of statin therapy in the treatment of human esophageal adenocarcinoma.

We thank Dr David Beer (Department of Surgery, University of Michigan, Ann Arbor, Mich) for his generous gift of the FLO-1 human esophageal adenocarcinoma cell line.

\section{References}

1. Brown LM, Devesa SS, Chow WH. Incidence of adenocarcinoma of the esophagus among white Americans by sex, stage, and age. J Natl Cancer Inst. 2008; 100:1184-7

2. Kubo A, Corley DA. Marked regional variation in adenocarcinomas of the esophagus and the gastric cardia in the United States. Cancer. 2002;95:2096-102.

3. Jankowski JA, Perry I, Harrison RF. Gastro-oesophageal cancer: death at the junction. BMJ. 2000;321:463-4

4. Jankowski JA, Harrison RF, Perry I, Balkwill F, Tselepis C. Barrett's metaplasia. Lancet. 2000;356:2079-85.

5. Lin YC, Shun CT, Wu MS, Chen CC. A novel anticancer effect of thalidomide inhibition of intercellular adhesion molecule-1-mediated cell invasion and metastasis through suppression of nuclear factor- $\kappa$ B. Clin Cancer Res. 2006;12: 7165-73.

6. Hayes SH, Seigel GM. Immunoreactivity of ICAM-1 in human tumors, metastases and normal tissues. Int J Clin Exp Pathol. 2009;2:553-60.

7. Roland CL, Harkin AH, Sarr MG, Barnett CC Jr. ICAM-1 expression determines malignant potential of cancer. Surgery. 2007;141:705-7.

8. Thommesen L, Sjursen W, Gåsvik K, Hanssen W, Brekke OL, Skattebøl L, et al. Selective inhibitors of cytosolic or secretory phospholipase $\mathrm{A}_{2}$ block 
TNF-induced activation of transcription factor nuclear factor- $\kappa \mathrm{B}$ and expression of ICAM-1. J Immunol. 1998;161:3421-30.

9. Huang WC, Chan ST, Yang TL, Tzeng CC, Chen CC. Inhibition of ICAM-1 gene expression, monocyte adhesion and cancer cell invasion by targeting IKK complex: molecular and functional study of novel $\alpha$-methylene- $\gamma$-butyrolactone derivatives. Carcinogenesis. 2004;25:1925-34.

10. Wu Y, Zhou BP. TNF- $\alpha / \mathrm{NF}-\kappa \mathrm{B} /$ Snail pathway in cancer cell migration and invasion. Br J Cancer. 2010;102:639-44.

11. Rockett JC, Darnton SJ, Crocker J, Matthews HR, Morris AG. Expression of HLA-ABC, HLA-DR and intercellular adhesion molecule-1 in oesophageal carcinoma. J Clin Pathol. 1995;48:539-44.

12. Agarwal B, Bhendwal S, Halmos B, Moss SF, Ramey WG, Holt PR. Lovastatin augments apoptosis induced by chemotherapeutic agents in colon cancer cells. Clin Cancer Res. 1999;5:2223-9.

13. Wu J, Wong WW, Khosravi F, Minden MD, Penn LZ. Blocking the Raf/MEK/ ERK pathway sensitizes acute myelogenous leukemia cells to lovastatininduced apoptosis. Cancer Res. 2004;64:6461-8.

14. Graaf MR, Richel DJ, van Noorden CJ, Guchelaar HJ. Effects of statins and farnesyltransferase inhibitors on the development and progression of cancer. Cancer Treat Rev. 2004;30:609-41.

15. Ogunwobi OO, Beales IL. Statins inhibit proliferation and induce apoptosis in Barrett's esophageal adenocarcinoma cells. Am J Gastroenterol. 2008;103:825-37.

16. Seljeflot I, Tonstad S, Hjermann I, Arnesen H. Reduced expression of endothelial cell markers after 1 year treatment with simvastatin and atrovastatin in patients with coronary heart disease. Atherosclerosis. 2002;162:179-85.

17. Hindler K, Cleeland CS, Rivera E, Collard CD. The role of statins in cancer therapy. Oncologist. 2006;11:306-15.

18. Shellman YG, Ribble D, Miller L, Gendall J, Vanbuskirk K, Kelly D, et al. Lovastatin-induced apoptosis in human melanoma cell lines. Melanoma Res. 2005; 15:83-9.

19. Konturek PC, Burnat G, Hahn EG. Inhibition of Barrett's adenocarcinoma cell growth by simvastatin: involvment of COX-2 and apoptosis-related proteins. $J$ Physiol Pharmacol. 2007;58(Suppl 3):144-8.

20. Boonstra JJ, van Marion R, Beer DG, Lin L, Chaves P, Ribeiro C, et al. Verification and unmasking of widely used human esophageal adenocarcinoma cell lines. J Natl Cancer Inst. 2010;102:271-4.

21. Sadaria MR, Meng X, Fullerton DA, Reece TB, Shah RR, Grover FL, et al. Secretory phospholipase $\mathrm{A}_{2}$ inhibition attenuates intercellular adhesion molecule-1 expression in human esophageal adenocarcinoma cells. Ann Thorac Surg. 2011; 91:1539-45. Epub 2011 Mar 10.

22. Mauchley D, Meng X, Johnson T, Fullerton DA, Weyant MJ. Modulation of growth in human esophageal adenocarcinoma cells by group Iia secretory phospholipase $\mathrm{A}_{2}$. J Thorac Cardiovasc Surg. 2010;139:591-9.

23. Meng X, Ao L, Song Y, Babu A, Yang X, Wang M, et al. Expression of functional Toll-like receptors 2 and 4 in human aortic valve interstitial cell: potential roles in aortic valve inflammation and stenosis. Am J Physiol Cell Physiol. 2008;294: C29-35.

24. Hachem C, Morgan R, Johnson M, Kuebeler M, El-Serag H. Statins and the risk of colorectal carcinoma: a nested case-control study in veterans with diabetes. Am J Gastroenterol. 2009;104:1241-8.

25. Kobayashi H, Boelte KC, Lin PC. Endothelial cell adhesion molecules and cancer progression. Curr Med Chem. 2007;14:377-86.

26. Mason RP. Molecular basis of differences among statins and a comparison with antioxidant vitamins. Am J Cardiol. 2006;98:34P-41P

27. Ahn KS, Sehti G, Aggarwal BB. Simvastatin attenuates TNF- $\alpha$-induced apoptosis through the down-regulation of NF- $\kappa \mathrm{B}-$ dependent antiapoptotic gene products: role of I $\kappa \mathrm{B} \alpha$ and TGF- $\beta$-activated kinase-1. J Immunol. 2007;178: 2507-16.

28. Nguyen DM, Richardson P, EI-Serag HB. Medication (NSAIDs, statins, proton pump inhibitors) and the risk of esophageal adenocarcinoma in patients with Barrett's esophagus. Gastroenterology. 2010;138:2260-6.

\section{Discussion}

Dr Raphael Bueno (Boston, Mass). Thank you for this elegant presentation and elegant work. It is always nicer when the drug that you discovered to have an effect in your cell line is approved by the Food and Drug Administration and any physician can justify giving it. So the question for you is, what is your next step? Are you going to add it to your postoperative regimen for patients or are you going to do something else to look into it? If this improves survival, why shouldn't I start giving this drug to everybody who has $\mathrm{T} 3 \mathrm{~N} 1$ disease after resection for esophageal cancer?

Dr Sadaria. That is an excellent question. The effects of simvastatin have been investigated in various other cancers, but in terms of esophageal adenocarcinoma, it has been researched only in vitro. I think the next step for us is to look at it in vivo, as you suggested, but probably in an animal model first to verify the doses used and to ensure no adverse effects at these doses. Depending on the results in an animal model, hoping, again, that it will inhibit tumor metastases without significant side effects, we will then plan to take it to the clinical level.

Dr Frank W. Sellke (Providence, RI). Did you look at other cell surface adhesion molecules to see whether they had similar effects?

Dr Sadaria. No, we have not evaluated other cell surface adhesion molecules. Thus far in our laboratory, we have only looked at the expression of ICAM-1. Now that we have demonstrated how ICAM-1 expression is attenuated with simvastatin therapy, one of the future directions we can take with this project is to look at other cell surface adhesion molecules and observe how they may be affected by statin therapy.

Dr Sellke. Why did you pick ICAM-1?

Dr Sadaria. ICAM-1 has actually been documented quite a bit as a strong contributor to the tumor's metastatic potential, more so in other tumor cell lines than in esophageal cancer. We recently published an article in regard to ICAM-1 and the metastatic potential of esophageal adenocarcinoma tumor cells. But again, we based our choice of ICAM- 1 on the fact that it has been widely documented in other tumor cell lines as being an important contributor in the tumor's metastatic potential. 